\title{
DER SONNENSTAND ALS ZEITGEBER FÜR DIE BIENE
}

\section{La position du soleil comme indicateur de temps chez l'Abeille}

\author{
Wilhelm BEIER 1 und Martin LINDAUER 2 \\ Zoologisches Institut der Technischen Hochschule, Darmstadt \\ Zoologisches Institut der Universität, Frankfurt am Main
}

\section{SUMMARY}

THE ADJUSTEMENT OF THE INTERNAL CLOCK OF HONEY BEES ACCORDING

\section{TO THE POSITION OF THE SUN}

1. The internal clock of honey bees can be reset, if time-training takes place in the field, the exogenous Zeitgeber, i. e. artificial light, however, has shifted only in a temperature conditioned room, where the hive stands.

None the less, an adjustment of the internal clock in field experiments is far more difficult than in the case, where both time-training and phase-shifting are achieved under laboratory conditions in a flight-room.

2. This fact is explained by the influence of the sun's position in the field. This position combined with land marks could be retained in the memory as a distinct pattern and could be used as a sundial $\%$.

3. Under laboratory conditions most time-trained bees arrive before the training-time (negative phase-shift). In field experiments, however, the maximum is observed at the beginning of or within the training time, if the sun is shining; on rainy or clowdy days the peak occurs also before the training time.

The more precise position of the maximum of arrivals in sunshine can also be explained by the additional synchronizing influence of a " sun-dial ".

4. Dawn and dusk do not influence the position of the collecting peak.

5. The same mechanism that regulates the time memory of bees and allows time-training, is responsible for time-compensated orientation according to the sun-compass orientation.

6. Sun-compass orientated bees do not use an average but a variable speed of the sun's azimut in order to compensate for the apparent change of the sun's position.

1, 2. Anschrift der Verfasser : Zoologisches Institut der Universität - 6 Frankfurt a. M. Sies• mayerstr. 70 - B.R.D. und Zoologisches Institut der Technischen Hochschule - 61 Darmstadt, Schnittspahnstr. 3 - B.R.D. 


\section{ZUSAMMENRASSUNG}

1. Eine Verstellung der inneren Uhr der Bienen gelingt auch dann, wenn die Zeitdressur im Freien, die Phasenverschiebung des exogenen Zeitgebers jedoch mit Kunstlicht im Klimaraum erfolgt. Gegenüber den Phasenverschiebungsversuchen mit Zeitdressuren im Flugraum läßt sich die innere Uhr bei Freilanddressuren deutlich schwerer verstellen.

2. Erklärt wird der stärkere Widerstand gegenüber dem phasenverschobenen künstlichen Licht-Dunkel-Zeitgeber im Freien durch den Einfluß des Sonnenstandes. Dieser könnte in Kombination mit Landmarken als festes " Muster " im Gedächtnis behalten und damit als "Sonnenuhr " benutzt werden.

3. Bei allen Flugraumdressuren liegt das Besuchsmaximum stets mehr oder weniger vor der Dressurzeit (negative Phasenwinkeldifferenz), bei den Freilandversuchen erscheint das Maximum an sonnigen Tagen erst zu Beginn oder in der Dressurzeit, an trüben, regnerischen Tagen jedoch ebenfalls vor der Dressurzeit.

Als Erklärung für die präzisere Lage des Besuchsmaximums an Sonnentagen wird wieder der zusätzlich synchronisierende Einfluß einer "Sonnenuhr" herangezogen.

4. Die Morgen- und Abenddämmerung hat keinen Einfluß auf die Lage des Besuchsmaximums.

5. Der gleiche Zeitmeßmechanismus, der dem Zeitgedächtnis der Bienen zugrunde liegt und eine Zeitdressur ermöglicht, ist auch für die Richtungsorientierung nach dem Sonnenkompaß verantwortlich.

6. Bei der Verrechnung der scheinbaren Sonnenwanderung für die Sonnenkompaßorientierung berücksichtigen die Bienen nicht eine mittlere, sondern die wechselnde Azimutwinkelgeschwindigkeit.

\section{A. - PRoblemstellung}

Jüngere Arbeiten über das Zeitgedächtnis der Bienen haben zwei wesentliche Ergebnisse erzielt :

1. Der Zeitmeßmechanismus kann experimentell (durch Narkose bzw. durch Phasenverschiebung) in mindestens 2 Komponenten zerlegt werden : die eine manifestiert sich als die starre circadiane Rhythmik, die andere läßt sich durch exogene Zeitgeber beeinflussen (Beier, Medugorac, Lindauer, 1968; BEIER, 1968).

2. Wie von anderen Autoren vielfach belegt, wird auch bei der Honigbiene der circadiane Rhythmus durch den tagesperiodischen Licht-Dunkel-Wechsel synchronisiert. Künstlicher Licht-Dunkel-Wechsel, der vom 24-Stunden-Intervall abweicht, vermag in begrenztem Ausmaß (von 10 bis 26 Std.) ebenfalls zu synchronisieren. Dabei gelingt bei einer Vorverlegung der Zeitgeberphase die Synchronisation schneller als bei einer Rückverlagerung. Der circadiane Rhythmus der Biene ist 23,4 Std. (Beier, 1968; vergl. auch Renner und Bennet, 1963).

Aus diesen Befunden erhebt sich zwingend die Frage nach der relativen Einflußgewalt von künstlichen und natürlichen Zeitgebern. Entscheidende Ansätze hierfür hatte bereits Renner in seinen Versetzungsversuchen erbracht (Renner, 1959 und 1960). Wir hofften in unseren Versuchen zu kłären, ob und inwieweit das Zeitgedächtnis der Bienen und ihre Sonnenkompaßorientierung auf dem gleichen ZeitmeBsystem beruhen. Diese Frage ist schon deshalb wichtig, weil trotz der Arbeiten von Grabensberger über Ameisen und Wespen (GraBENSBERGER, 1934 $a, b, c$ ) ein Zeitgedächtnis bei Insekten exakt nur für Bienen nachgewiesen werden konnte (BELING, 1929; WAHL, 1932 und 1933; RENNER, 1958). Demgegenüber scheint die Fähigkeit zur Sonnenkompaßorientierung nahezu Allgemeingut der Arthropoden zu sein (v. Frisch, 1965 und Lindauer, 1963). 


\section{B. - MATERIAL UND METHODE}

Wie in vorausgegangenen Untersuchungen (Beier, 1968, und Beier, Medugorac, Lindauer, 1968), erfolgte die Synchronisation der inneren Uhr der Bienen durch den künstlichen Licht-DunkelZeitgeber in einem Bienenflugraum.

Bei den kombinierten Flugraum-Freilandversuchen hatten die Versuchstiere außerdem die Möglichkeit, durch eine Schleuse ins Freie zu gelangen. Die Kontrolle des Ausflugs und vor allem der Ausflugszeit aus dem Bienenflugraum ins Freie, bzw. der im Freien aufgestellten Völker, wurde durch ein mit leichten Kuntstoffklappen gesteuertes "Einbahnsystem " vorgenommen. (vergl. BEIER, 1968, Abb. 2).

Bei der Verstellung der inneren Uhr der Bienen wurde die verschobene Licht-Dunkel-Phase zur längeren und besseren Einwirkung unter simulierten Schlechtwetterbedingungen im Klimaraum bei $+8{ }^{\circ} \mathrm{C}$ geboten. Die Bienen fliegen bei dieser Temperatur nicht aus, andererseits läßt sich die innere Uhr durch den gebotenen Lichtrhythmus einer Glasscheibe auch im Stock direkt ohne Schwierigkeiten steuern (vergl. auch hierzu die ausführliche Darstellung in der Arbeit von BEIER, 1968, S. 359).

Die Dressur auf eine bestimmte Tageszeit und die Registrierung der Versuchstiere erfolgten prinzipiell nach den gleichen Methoden wie sie von BeLing, 1929, WaHL, 1932, und von Frisch, 1921, erarbeitet wurden. Notwendige Änderungen (vergl. BEIER, 1968, S. 357) dienten vor allem zur schärferen Erfassung der Genauigkeit der inneren Uhr.

In nahezu allen Versuchen wandten wir die individuelle Registrierung durch Direktbeobachtung an; lediglich aus dem Flugraum wurden einige Versuche mit nicht individueller Registrierung (vergl. RENNER, 1959) zum Vergleich herangezogen.

\section{C. - PHASenverschiebung DURCH KÚNSTLICHEN LICHT-DUNKEL-WECHSEL}

\section{1. - Einmalige Phasenverschiebung des Licht-Dunkel-Wechsels}

Der Einfluß potentieller exogener Zeitgeber auf die innere Uhr der Bienen wird im hermetisch abgeschlossenen Flugraum bei konstanter Temperatur und Luftfeuchtigkeit auf den künstlichen Licht-Dunkel-Wechsel reduziert. Um den natürlichen Sonnentag in Konkurrenz mit einem künstlich gesetzten LichtDunkel-Wechsel zu setzen, werden die Bienen zwar im Flugraum gehalten, dürfen aber während der Lichtzeit ins Freie ausfliegen, und hier erfolgt auch die Dressur auf eine bestimmte Futterzeit. Der künstliche Licht-Dunkel-Wechsel entspricht dem natürlichen unter Wegfall der Dämmerung. Ein erster Kontrollversuch (Abb. 1a) zeigt, daß die Bienen auch ohne Dämmerung zur genauen Dressurzeit erscheinen. Künstlicher und natürlicher Tag stören sich also nicht; sie wirken synchron auf das Zeitgedächtnis. Jetzt führen wir im lichtdicht abgeschlossenen Raum eine Phasenverschiebung des künstlichen Licht-Dunkel-Wechsels durch. Aus einer vorhergehenden Arbeit (BEIER, 1968) wissen wir, daß durch diese Phasenverschiebung die innere Uhr der Bienen in 3 bis 4 Tagen verstellt wirdauch wenn die Dressur unter Ausschluß der Sonnensicht erfolgte. Abb. 1 bringt Verlauf und Ergebnis unseres Versuches. Die Licht-Dunkel-Periode wurde nach dem Vergleichsversuch durch Verlängerung um 5 Std. phasenverschoben. Diese Phasenverschiebung wurde 7 Tage hintereinander in dem Sinne wiederholt, daß " Licht an " statt auf 6 Uhr auf 11 Uhr fiel. Die Bienen durften zur Verhinderung der Abdressur (vgl. BeIER, 1968, S. 359) in den ersten 3 Tagen nicht ausfliegen und, was wichtig ist, auch nicht den natürlichen Tag sehen. Am 4. Tag wurde der Ausflug um 11 Uhr freigegeben. 


\section{Aвв. 1. - Zeitdressur und Beobachtung an den Versuchstagen im Freien}

Verstellung der inneren Uhr der Bienen durch Phasenverschiebung des künstlichen Licht-DunkelWechsels im Flugraum. Dem Vergleichsversuch (a) folgen zwei Dressurtage und drei Tage Arretierung bei verstellter Licht-Dunkel-Zeit $(+5$ Std.). Die Bienen bekommen während dieser drei Tage den natürlichen Tag nicht zu sehen. An den folgenden vier Beobachtungstagen (b-e) dürfen die Bienen ab $11 \mathrm{Uhr}$ frei ausfliegen. Am Abend wird ihnen die natürliche Dämmerung nicht verwehrt.

\section{FIg. 1. - Dressage au temps et observations effectuées} pendant les journées d'expériences en plein air

Décalage de l'horloge interne des abeilles par déphasage de l'alternance artificielle lumièreobscurité dans la chambre de vol. L'expérience témoin (a) est suivie de deux jours de dressage et trois jours de claustration par temps décalé lumière-obscurité ( +5 heures). Pendant ces trois jours les abeilles ne voient pas le jour naturel. Pendant les quatre jours d'observation suivants (b-e) les abeilles pourront voler librement à partir de 11 heures. Le crépuscule naturel ne leur sera pas interdit.

Anzahl der suchenden Bienen : nombre d'abeilles butineuses.

Beobtg : observation.

Zeit : temps (l'heure).

Vergleichsversuch : expérience témoin.

Lichtzeit : temps d'éclairage.

Phasenversch. Lichtzeit : temps d'éclairage déphasé. 

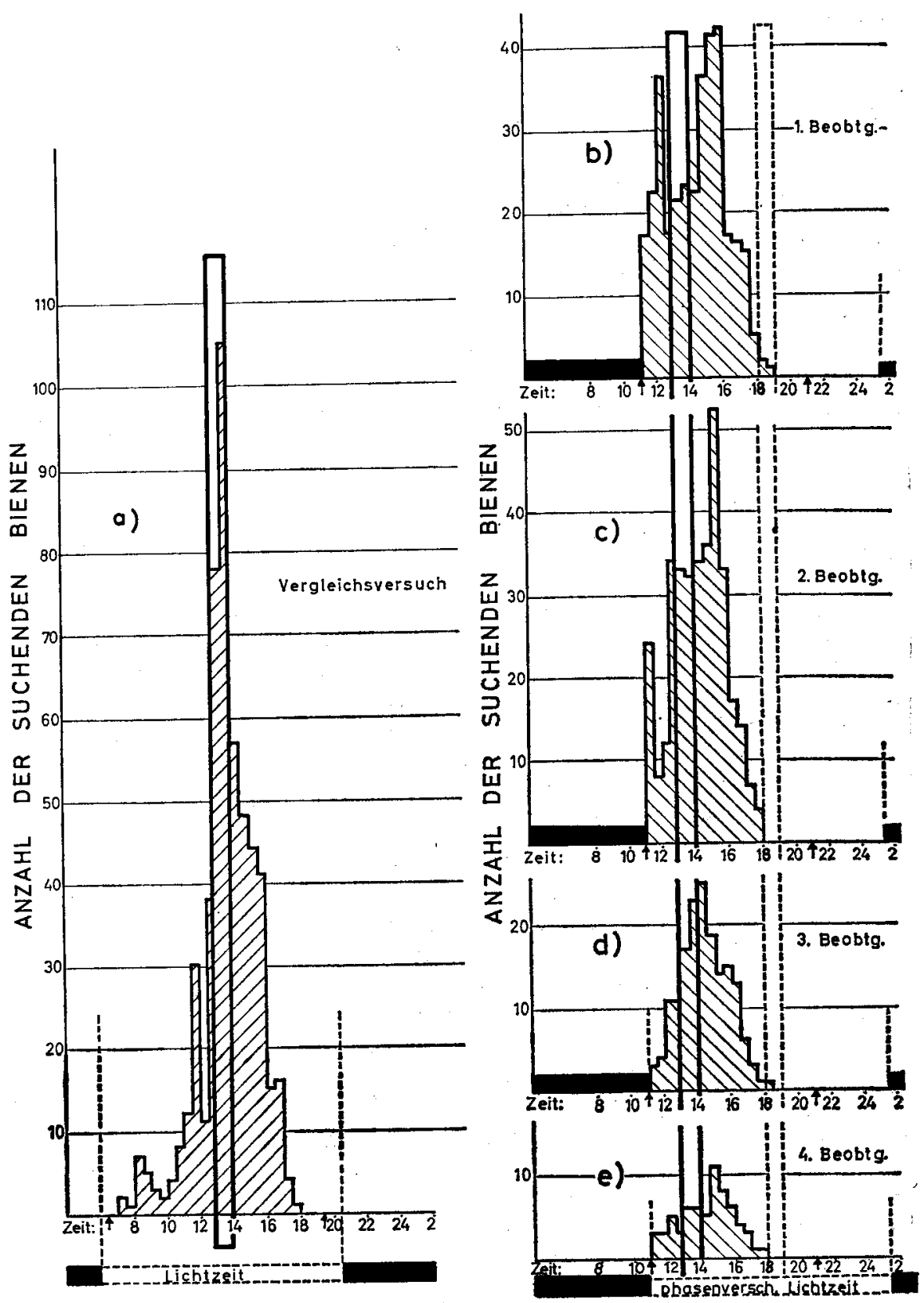

Abb. 1. - Fig. 1. 
Die spontanen Besuche am Futtertisch im Freien vom 4. bis 7. Tag zəigen einerseits die gewohnte Dissoziation nach der Phasenverschiebung, andererseits auch eine deutliche Verlagerung des zweiten und damit exogenen Maximums um etwa 2 Std.. Dieser Wert liegt aber klar unter den vergleichbaren Verstellungen bei Ausschluß von Sonnensicht (BeIER, 1968, S. 362). Im letzten Fall war die Verstellung nach 3 bis 4 Tagen vollständig, und die Bienen kamen genau zur fiktiven Dressurzeit. Bemerkenswert sind die Ergebnisse an den folgenden 3 Tagen: die Verschiebung der Besuche am Futtertisch schreitet nicht zugunsten des künstlichen Tages fort; im Gegenteil, das Maximum wandert eher wieder in Richtung auf die alte Dressurzeit zurück: ungefähr um 1/2 Std. am 2. und nochmals um 1 Std. am 3. Beobachtungstag. Und das, obwohl der vorher umstellend wirkende Licht-Dunkel-Wechsel bestehen bleibt!

Welche tagesperiodischen Abłäufe haben diese Rückverschiebung bewirkt? Aufgrund früherer Untersuchungen von BELING (1929), WAHL (1932) und ReNNER (1959) und zahlreicher eigener Kontrollen scheiden Schwankungen der Temperatur und der relativen Luftfeuchtigkeit als wirksame äußere Zeitgeber aus. Die abendliche Dämmerung und ein evtl. Umstellen der Bienen auf einen verkürzten natürlichen Tag können ebenfalls nicht beteiligt sein, da die Bienen bereits am ersten Beobachtungstag im Freien ein zu frühes Maximum bringenund zwar bevor sie überhaupt das Ende dieses ersten "verkürzten Naturtages" erfuhren.

Gegen einen möglichen Einfluß der abendlichen Dämmerung bringt ein weiterer Versuch Belege : Wenn man die Ausflugzeit der Bienen so beschränkt, daß sie weder während der Dressur noch während der Beobachtung eine Morgenoder Abenddämmerung erleben, so erhält man das Ergebnis von Abb. 2. Neben der gewohnten Dissoziation ergibt sich wiederum eine Verstellung um 2 1/4 Std. und ein Zurückwandern des exogenen Maximums am 2. und 3. Beobachtungstag. Es muß hinzugefügt werden, daß bei beiden Versuchen (Abb. 1 und 2) warmes, sonniges Sommerwetter herrschte; lediglich am ersten Beobachtungstag war der Himmel beide Male bis gegen $16 \mathrm{Uhr}$ bzw. bis gegen $12 \mathrm{Uhr}$ mit Wolken verhangen.

Es war noch zu prüfen, ob sich die innere Uhr der Bienen auch durch eine Vorverschiebung der Zeitgeberphase einer Verstellung stärker widersetzt, wenn die Zeitdressur im Freien erfolgt. Es ist bekannt, daß die circadiane Periodenlänge der Biene 23,4 Std. beträgt (BEIER, 1968). Der exogene Zeitgeber und die endogene Komponente des ZeitmeBmechanismus wirken in dem geplanten Versuch in einer Richtung. Wird die innere Uhr solcher Synchronisation leichter folgen? Abb. 3 bestätigt diese Vermutung. Obwohl schon am zweiten Tag nach der Phasenverschiebung ein Test eingelegt wurde, kamen die Bienen gegenüber dem Vergleichsdiagramm wiederum 2 Std. früher zum Futterplatz. An den beiden folgenden Beobachtungstagen verschiebt sich dann das Besuchsmaximum weiter auf die fiktive Dressurzeit zu. Allerdings beträgt die zeitliche Verschiebung der Maxima jeweils nur noch 1/2 Std. Erst am 4. Beobachtungstag verschiebt sich das Maximum wieder wie in den früheren Versuchen auf die alte Dressurzeit zurück.

Eine Phasenverschiebung durch Vorverlegung der Licht-Dunkel-Phase führt demnach leichter zum Erfolg als durch eine entsprechende Rückverlegung. Aber auch in diesem Fall wird dem künstlichen Licht-Dunkel-Zeitgeber im Freien 

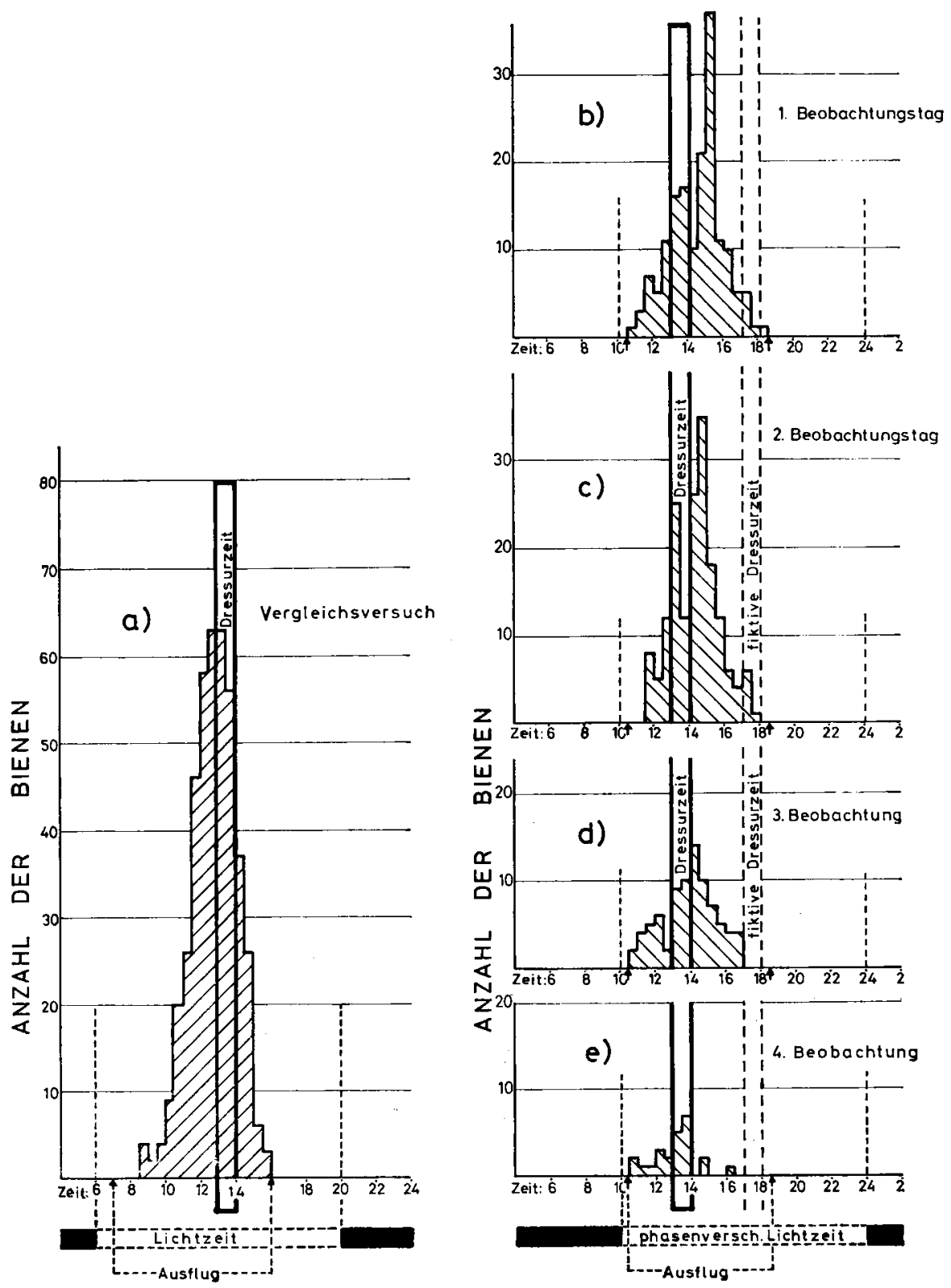

Aв8. 2. - Freilandversuch mit Phasenverschiebung des künstlichen Licht-Dunkel-Wechsels im Flugraum (vergl. Legende zu Abb. 1)

Hier in Abb. 2 wird jedoch den Bienen mit Hilfe von Klappendurchgängen am Stock (vergl. BEIER, 1968) die Ausflugzeit morgens und abends kontrolliert eingeengt. Verschiebung des LichtDunkel-Wechsels um +4 Std.

Fig. 2. - Expérience en plein air avec déphasage de l'alternance artificielle lumière-obscurité dans la chambre de vol (voir légende de la figure 1)

Ici on réduit le temps de sortie des abeilles, matin et soir, en le contrôlant à l'aide des portillons de la ruche (voir BEIER, 1968). Déphasage de l'alternance lumière-obscurité de +4 heures.

Dressurzeit : temps de dressage.

Fiktive Dressurzeit : temps fictif de dressage Beobachtungstag (Beobachtung) : jour d'observation. Ausflug : sortie. 

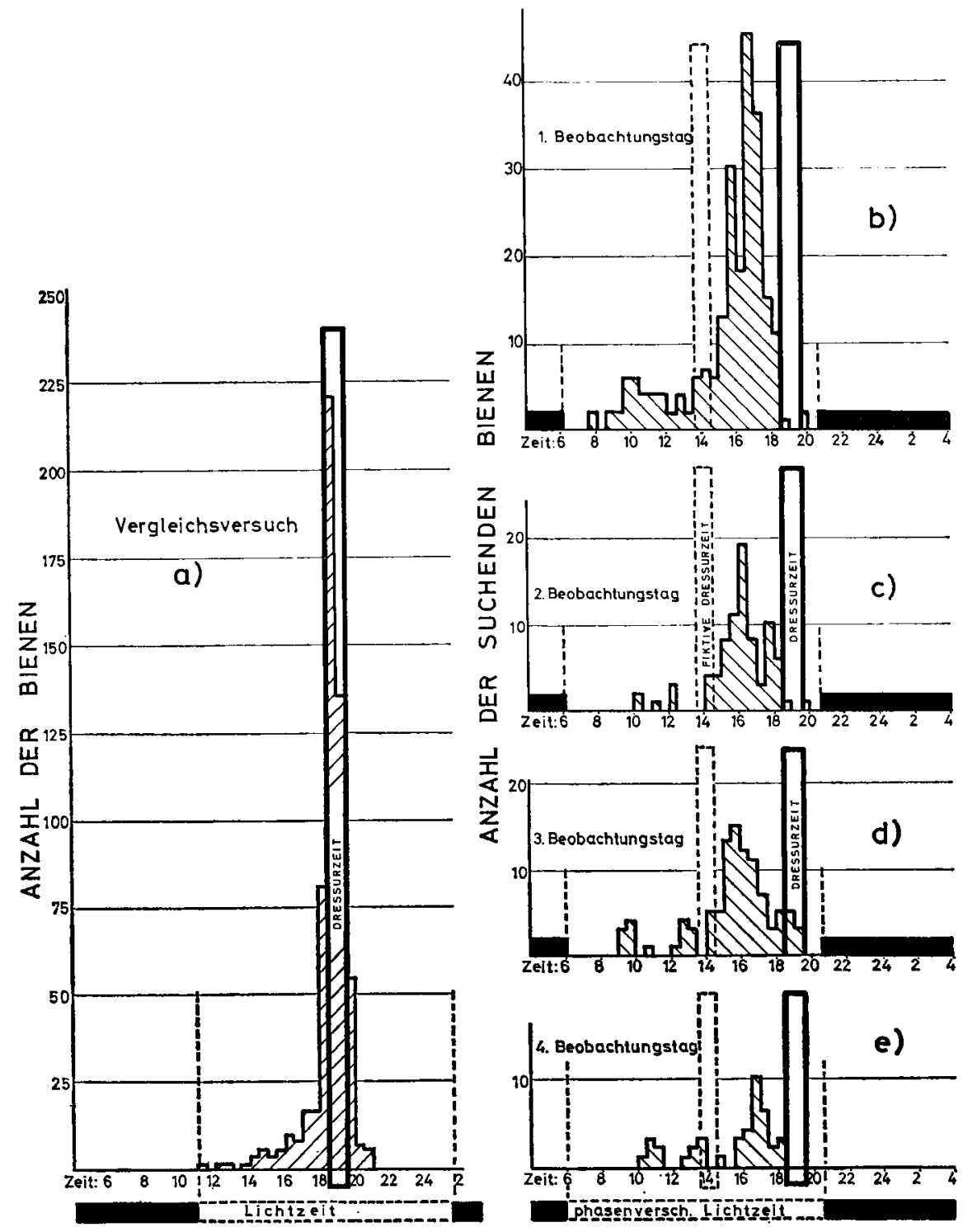

Авв. 3. - Freilandversuch mit Phasenverschiebung des künstlichen Licht-Dunkel-Wechsels im Flugraum um - 5 Stunden

Nach dem Vergleichsversuch (a) zwei Dressurtage. Dann anschließend ein Tag Arretierung im Flugraum mit phasenverschobenem Licht-Dunkel-Wechsel und vier Beobachtungstagen. Die abendliche Dämmerung wird nicht verwehrt.

FIG. 3. - Expérience en plein air avec dêphasage de l'alternance artificielle lumière-obscuritế en chambre de vol de -5 heures

Après l'expérience témoin (a) deux jours de dressage. Ensuite, en incluant un jour d'arrêt dans la chambre de vol avec l'alternance lumière-obscurité déphasée et quatre jours d'observation. Le crépuscule ne sera pas interdit. 
ein erheblicher Widerstand durch einen exogenen Faktor des natürlichen 24Stunden-Tages entgegengesetzt.

\section{2. - Dressur auf einen 22-Stunden-Tag mit Freilandfütterung}

Wir variieren unsere Fragestellung noch einmal, indem wir den natürlichen Sonnentag (ohne Morgen - und Abenddämmerung) mit dem künstlichen 22Stunden-Tag in Konkurrenz setzen. Nach BEIER (1968) gelingt ja die Umstellung auf einen 22-Stunden-Tag ohne Schwierigkeit.

Das Ergebnis ist in Abb. 4 dargestellt. Deutlich tritt an beiden Beobachtungstagen ein Maximum zur 22-Stunden-Dressurzeit auf, aber auch der Einfluß des natürlichen Tages ist unverkennbar : 24 Std. nach der letzten Fütterung bzw. nach dem Hauptmaximum des ersten Beobachtungstages kommt es zu einem auffälligen Anstieg der Besuchsfrequenz. Man könnte mit Recht einwenden, daß dieses zweite Maximum durch den endogenen circadianen Rhythmus geprägt sei, dessen Periodenlänge nicht allzu weit von 24 Std. entfernt liegt $(23,4$ Std.). Die entsprechenden Phasen im Flugraumversuch sind jedoch nicht so ausgeprägt wie hier im Freien. Außerdem ist ein drittes Maximum, das der Fütterung vor 48 Std. entspricht, zu erkennen.

Zusammengefaßt : Bei Phasenverschiebung im Flugraum und gleichzeitiger Dressur im Freiland wird die innere Uhr der Bienen einer Konfliktsituation ausgesetzt; die Verschiebung der Besuchsmaxima erfolgt im Freien von Tag zu Tag langsamer als im Flugraum, oder die Maxima schieben sich sogar allmählich auf die alte Dressurzeit zurück. Unser Verdacht, die Sonne wäre der entscheidende Gegenspieler beim Phasensprung, verdichtete sich immer mehr. Ehe wir die Frage vorlegen, ob Azimut oder Sonnenhöhe oder andere Parameter Einfluß nehmen, sei eine weitere Beobachtung über die Synchronisationswirkung der Sonne mit dem 24-Stunden-Rhythmus vorausgeschickt.

\section{D. - ANALYSE DER PHASENWINKELDIFFERENZ}

Normale Zeitdressuren im Flugraum mit künstlichem Licht-Dunkel-Wechse brachten ausnahmslos ein Maximum, das vor der Dressurz it lag (vgl. BEIER, 1968, Abb. 4, S. 361, Vergleichsdiagramm). Die Phasenwinkeldifferenz (in Anlehnung an Aschoff und Wever, 1966) zwischen Besuchsmaxima und Dressurzeitbeginn war also streng negativ. Nicht so bei den vergleichbaren Versuchen im Freiland. Hier lagen nämlich die Maxima der meisten Zeitdressuran innerhalb der Dressurzeit, wiesen also eine positive Phasenwinkeldifferenz auf (Abb. 3, Vergleichsdiagramm). In Abb. 5 wird der Unterschied beider Gruppen in bezug auf die zeitliche Lage ihrer Maxima verdeutlicht. Für die Flugraumversuche ergibt sich danach eine klare Kulmination aller Besuchsmaxima 35 Min. vor der Dressurzeit. Das Ergebnis bleibt das gleiche, wenn man dem Freilandvolk núr begrenzte Ausflugzeit (10 bis $17 \mathrm{Uhr}$ ) bietet, um eine evtl. Dämmerungssynchronisation auszuschalten (Abb. 6).

Eine Prüfung beider Gruppen auf Homogenität ergibt für die Flugraumversuche eine homogene Gruppe, für die Freilandversuche nicht. Wir haben daher 

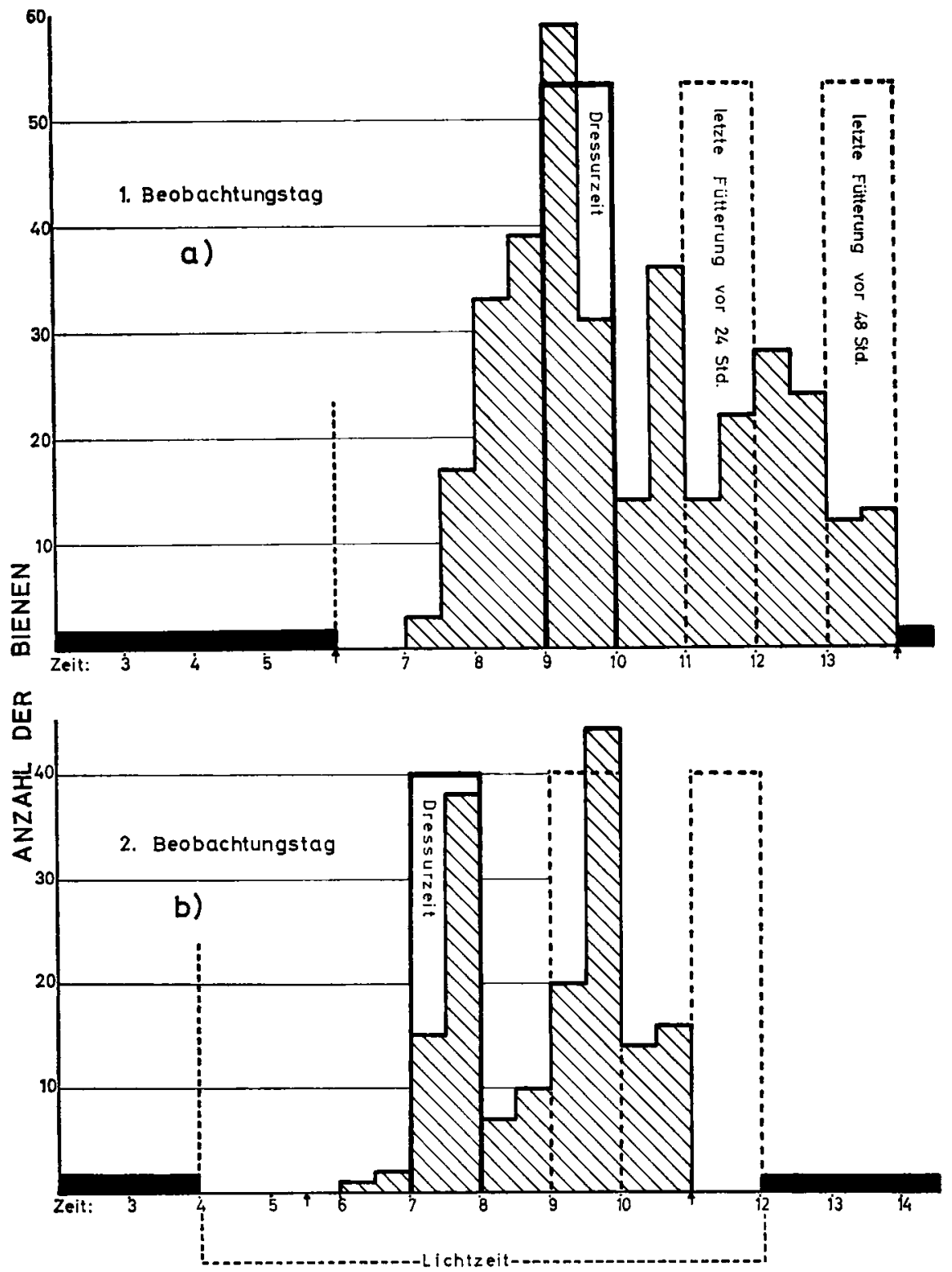

Aвв. 4. - Dressur auf einen 22-Stunden-Rhythmus

Die Bienen können nur während der Lichtzeit aus dem Flugraum ausfliegen. Der Futterplatz befindet sich im Freien $180 \mathrm{~m}$ vom Stock entfernt, der im Flugraum steht.

Fig. 4. - Dressage sur un rythme de 22 heures

Les abeilles ne peuvent sortir de la chambre de vol que pendant qu'il fait jour. La source de nourriture se trouve à l'extérieur, à $\mathbf{1 8 0}$ mètres de la ruche qui est placée dans la chambre de vol.

Letzte Fütterung vor 48 Std : dernier nourrissement 4.8 heures plus tòt. 


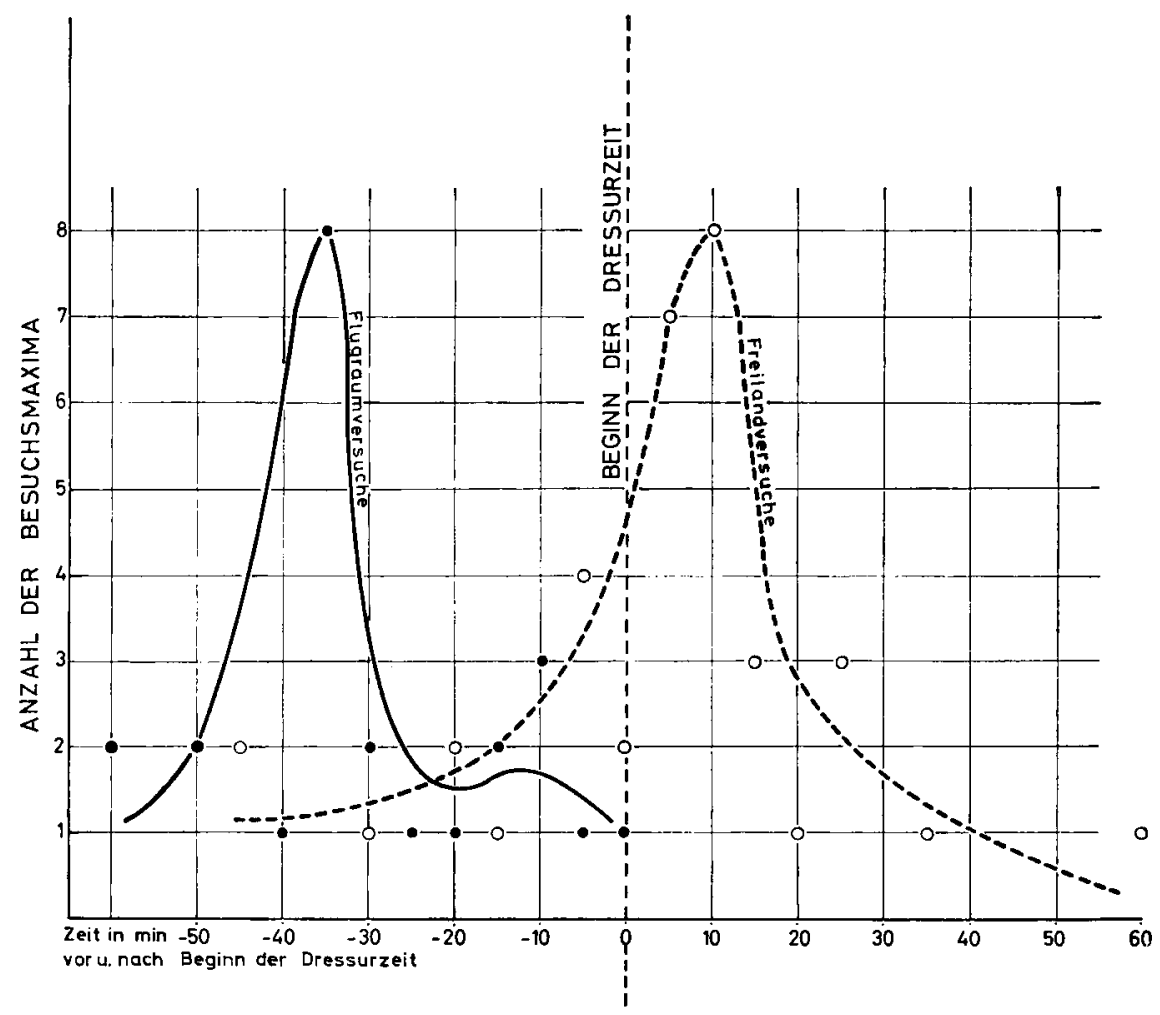

Авв. 5. - Gegenüberstellung vergleichbarer Zeitdressurversuche im Flugraum und im Freiland in bezug auf die zeitliche Lage ihrer Maxima

Auf der Abszisse ist die Zeit in Minuten vor und nach Beginn der Dressurzeit eingetragen. Schwarze Kreise = Maxima der Flugraumversuche; weiße Kreise = Maxima der Freilandversuche.

FIc. 5. - Confrontation d'expériences comparables de dressage au temps, en chambre de vol et à l'extérieur, en rapport avec la position temporelle des maxima

En abscisses est porté le temps en minutes avant et après le début du temps de dressage. Cercles noirs : maxima des expériences en chambre de vol. Cercles blanes : maxima des expériences à l'extérieur.

Anzahl der Besuchsmaxima : nombre des maxima de visites.

Flugraumversuche : expériences en chambre de vol.

Beginn der Dressurzeit : début du temps de dressage.

Freilandversuche : expériences en plein air.

Zeit in min vor u. nach Beginn der Dressurzeit : temps en minutes avant et après le début du temps de dressage. 


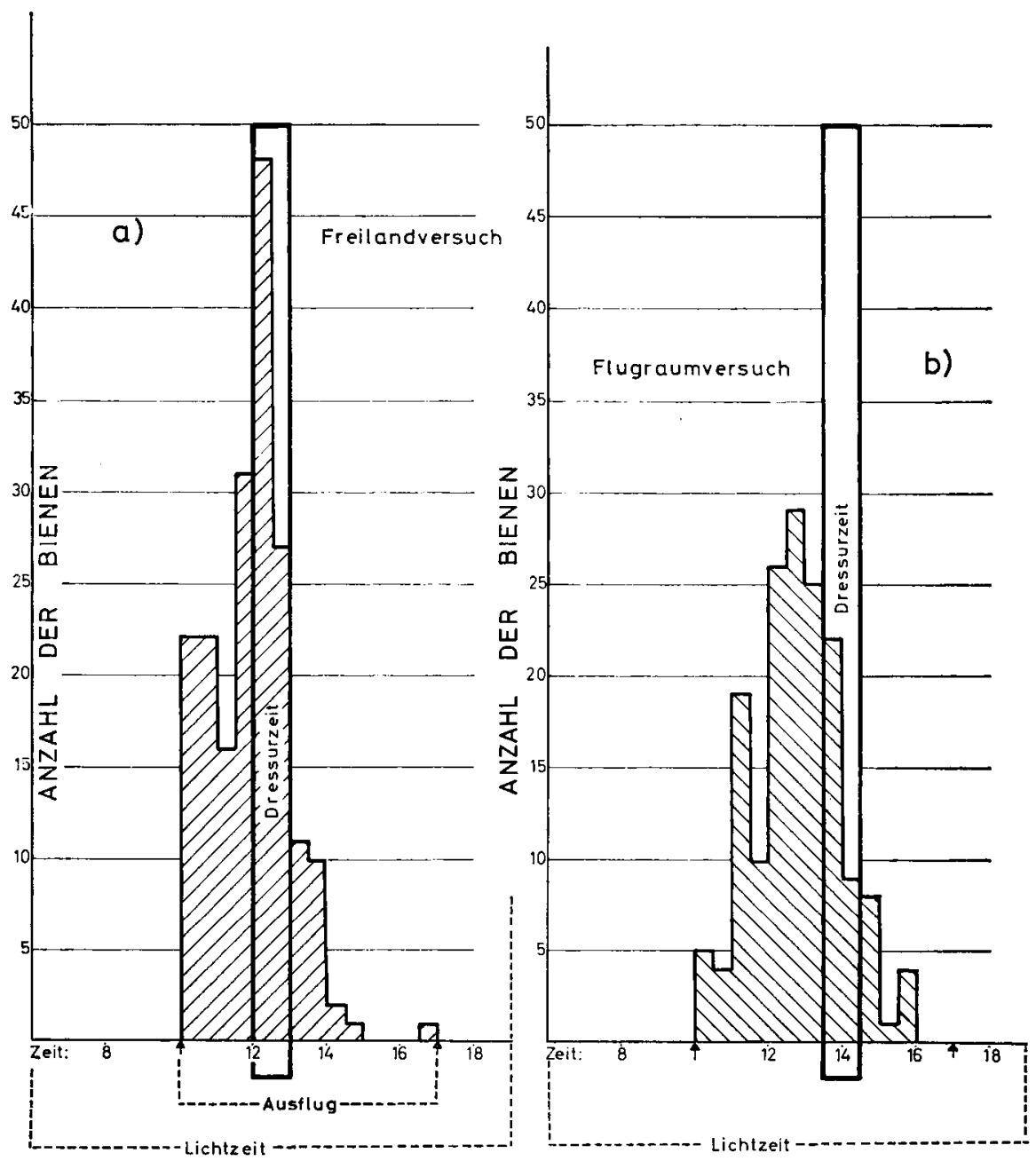

Aвв. 6. - Freiland- und Flugraumversuch zur Feststeliung des Dämmerungseinflusses auf das Zeitgedächtnis der Bienen

Die Versuchsscharen stammen aus zwei Völkern, die beide im Flugraum die gleiche Lichtzeil haben ( $\mathrm{L}: \mathrm{D}=13$ : 11). Nur das erste Volk $(a)$ hat jedoch die Möglichkeit, durch einen langen Gang zu einer begrenzten Zeit zwischen 10 und $17 \mathrm{Uhr}$ ins Freie auszufliegen.

Fıc. 6. - Expérience à l'extêrieur et en chambre de vol montrant l'influence de l'aurore et du crépuscule sur la mémoire du temps chez l'abeille

Les groupes d'ouvrières proviennent de deux colonies qui ont toutes deux le même temps d'éclairement dans la chambre de vol (lumière : obscurité $=13: 11$ ). Seule la première colonie $(a)$ a toutefois la possibilité de s'envoler à l'extérieur par un long couloir, pendant un temps limité compris entre 10 et 17 heures.

Ausflug : sortie. 
die Gesamtzahl der Freilandversuche nach verschiedenen Gesichtspunkten in Gruppen untergliedert (Tab. 1). Eine deutliche Korrelation ergab sich für Versuche bei sonnigem Wetter oder aufgelockerter Bewölkung und bei solchen mit regnerischem Wetter und völlig bedecktem Himmel.

TaB. 1. - Anflugmaxima im Flugraum gegenübergestellt den Maxima im Freien

a. Bei sonnigem - b. Bei bedecktem Himmel

TABL. 1. - Maxima des vols à l'intérieur, comparés aux maxima en plein air

a. Par temps ensoleillé - b. Par temps couvert

\begin{tabular}{|c|c|c|c|}
\hline & $\begin{array}{c}\text { Zahl der } \\
\text { Vers. } \\
\text { Nombre } \\
\text { d'expér. }\end{array}$ & $\begin{array}{l}\text { Max. vor d. } \\
\text { Dressurzeit } \\
\text { Max. avant } \\
\text { 1'heure } \\
\text { du dressage }\end{array}$ & $\begin{array}{l}\text { Max. in d. } \\
\text { Dressurzeit } \\
\text { Max. pendant } \\
\text { l'heure } \\
\text { du dressage }\end{array}$ \\
\hline $\begin{array}{l}\text { Flugraumversuche } \ldots \ldots \ldots \ldots \ldots \ldots \ldots \ldots \ldots, \ldots \ldots \ldots \\
\text { Expér. chambre de vol. }\end{array}$ & 24 & $24(100 \%)$ & - \\
\hline $\begin{array}{l}\text { Alle Freilandversuche } \ldots \ldots \ldots \ldots \ldots \ldots \ldots \ldots \ldots \ldots \\
\text { Ensemble exper. plein air. }\end{array}$ & 36 & $11(31 \%)$ & $25(69 \%)$ \\
\hline $\begin{array}{l}\text { Freilandversuche mit Sonne } \ldots \ldots \ldots \ldots \ldots \ldots \ldots \ldots \ldots \\
\text { Exper. plein air, temps ensoleillé. }\end{array}$ & 26 & $2 \quad(8 \%)$ & $24(92 \%)$ \\
\hline $\begin{array}{l}\text { Freilandversuche b. bedeckt. Himmel. . . . . . . . } \ldots \ldots \\
\text { Expér. plein air, temps couvert. }\end{array}$ & 10 & $9(90 \%)$ & $1(10 \%)$ \\
\hline
\end{tabular}

Deutlich tritt bei fehlender Sonne auch im Freien eine Desynchronisation des endogenen Rhythmus ein.

Auf vor auf eine negative Phasenwinkeldifferenz in bezug auf den Dressurzeitbeginn haben schon BeLING (1929), WAHL (1932) und RenNer (1957) hingewiesen. In keinem Fall erkannte man darin eine absolute Unschärfe des Zeitgedächtnisses; man sah in der Differenz vielmehr eine biologische Sinnfälligkeit, denn es ist ja vorteilhafter für eine Sammelschar, etwas früher als zu spät am Futterplatz zu erscheinen. Damit läßt sich aber die unterschiedliche Lage der Besuchsmaxima zwischen unseren Flugraumversuchen und Freilandversuchen nicht erklären. Die Sonne wirkt bei Dressur im Freien als exogener Synchronisator. Sie ist eine zusätzliche "Zeitmarke " im Sinne einer "Sonnenuhr ". 
W. BEIER, M. LINDAUER

\section{Авв. 7. - Fächer- und Versetzungsversuch}

a. Vergleichsversuch in einem großen Wiesengelände bei Offenthal. Zehn Beobachter sind von $0^{\circ}$ über $90^{\circ}$ bis $270^{\circ}$ fächerförmig mit einem Radius von $180 \mathrm{~m}$ um den Stock postiert. Dressurrichtung ist Süd $\left(180^{\circ}\right)$. Jeder Kreis bedeutet den Anflug von 5 individuell markierten Bienen am Beobachtungstischchen. Die Pfeile deuten die Windrichtung an den Versuchstagen an.

b. Drei Tage lang im Klimaraum Verstellung der Licht-Dunkel-Phase um -5 Std. Danach Beobachtung nach einer Versetzung über $5 \mathrm{~km}$ in die Nähe von Götzenbain. Ähnliches Wiesengelände wie bei $a$; wolkenverhangener Himmel.

\section{Fig. 7. - Expérience de l'éventail et du déplaccment}

a. Expérience témoin dans une grande prairie près d'Offenthal. Dix observateurs sont postés autour de la ruche, dans un éventail de 0 à $270^{\circ}$ en passant par $90^{\circ}$ et dans un rayon de $180 \mathrm{~m}$. La direction du dressage est le sud $\left(180^{\circ}\right)$. Chaque cercle représente l'envol de cinq abeilles, individuellement marquées, vers les tablettes d'observation. Les flèches montrent la direction du vent le jour de l'observation.

b. Pendant trois jours dans la chambre elimatisée le décalage de la phase lumière-obscurité est de -5 heures. Ensuite, observation après un déplacement de $5 \mathrm{~km}$, à proximité de Götzenhain. Même genre de prairie que pour $a$ et ciel nuageux.

Beobachtungstag nach der Verstllg. $d$. i. Uhr : jour d'observation après le décalage de l'horloge interne. 


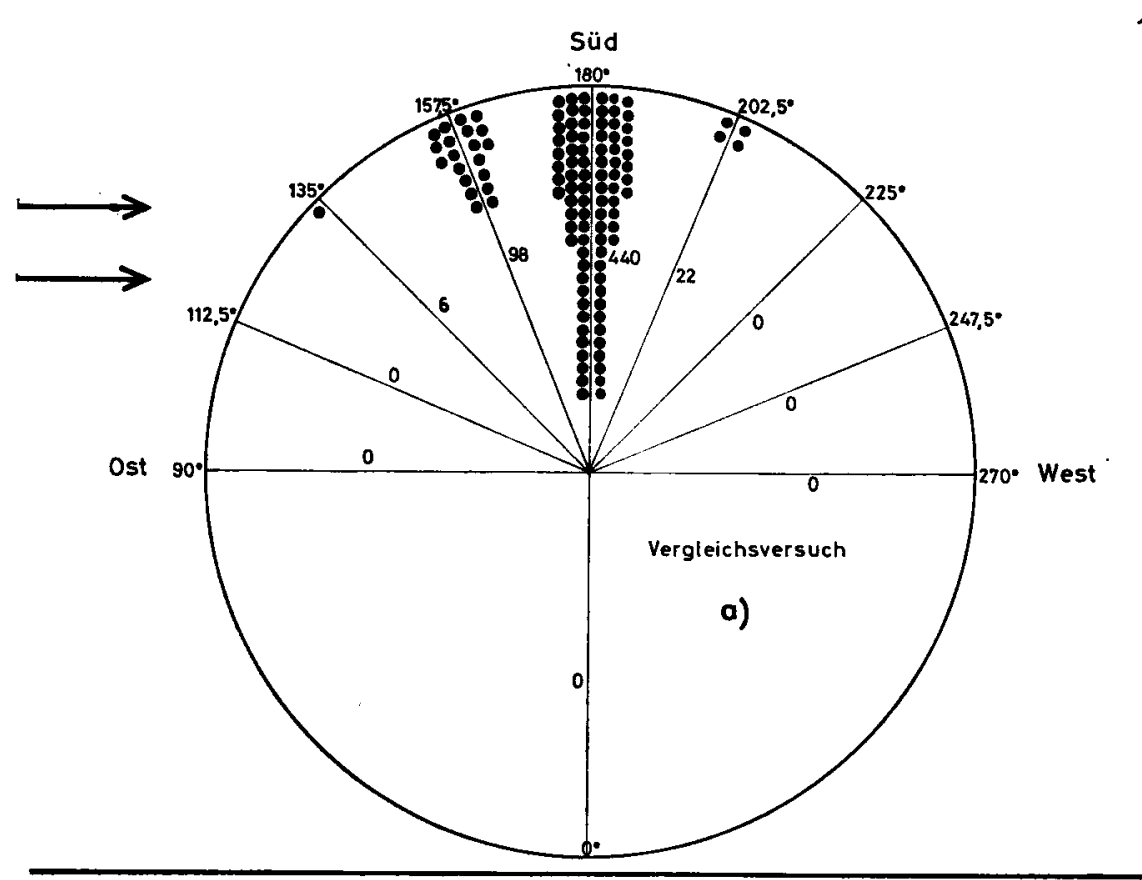

$\times 0,5$.

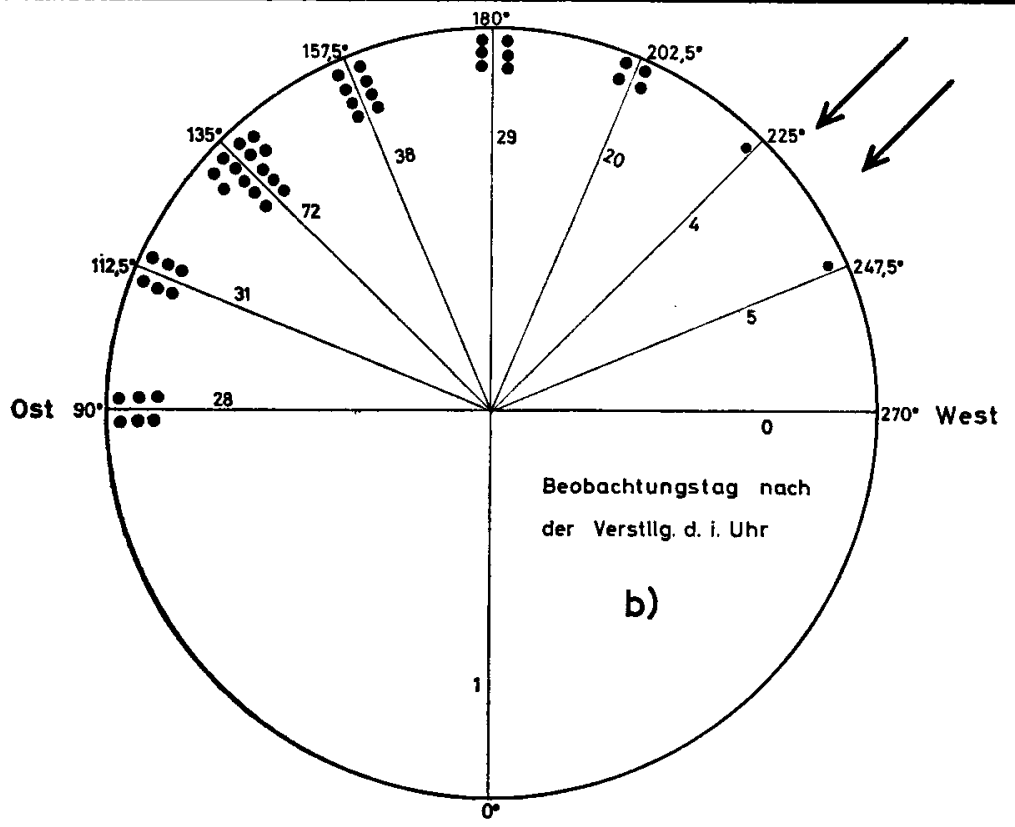




\section{E. - ZEITGEDÄCHTNIS UND SONNENKOMPASSORIENTIERUNG}

Wir wollen jetzt in einem geeigneten Versuch eine klare Aussage erzwingen, inwieweit der Sonnenstand bei der Synchronisation einer durch Phasensprung verstellten inneren Uhr der Biene beteiligt ist.

Aus Versetzungsversuchen ist bekannt (v. Frisch und Lindauer, 1954), daB Bienen nach Richtungsdressur und anschließender Versetzing in fremdes Gelände ihr Futterziel allein mit Hilfe des Sonnenkompasses aufsuchen. Dazu brauchen sie erstens eine genaue Uhrzeit, zweitens eine genaue Kenntnis des jeder Tageszeit entsprechenden Sonnenstandes.

Wir modifizieren diesen Versetzungsversuch, indem wir den richtungsdressierten Bienen eine falsche Uhrzeit mitgeben. Auf einem $1 \mathrm{~km}^{2}$ großen Wiesenge. lände ohne auffällige Landmarken dressieren wir eine Bienenschar auf die Zeit von $16.15 \mathrm{Uhr}$ bis $17.15 \mathrm{Uhr}$. Der Futterplatz ist $180 \mathrm{~m}$ südlich vom Stock. Von 12.00 Uhr bis 17.30 Uhr können die Bienen frei ausfliegen; anschließend kommen sie in den Bienenraum zurück, wo der künstliche Tag bis $24.00 \mathrm{Uhr}$ andauert (Hellzeit von 11.00 Uhr bis 24.00 Uhr). Nach 5 Dressurtagen erfolgt die Kontrolle der Zeit- und Richtungsdressur. 10 Beobachter registrieren die Anflüge an leeren Futtertischchen, die mit Radius $180 \mathrm{~m}$ fächerförmig um den Stock angeordnet sind (Abb. 7). Das Besuchsmaximum für alle Beobachtungsplätz? liegt in der Dressurzeit (Abb. 8a). Wie erwartet, wird auch die Dressurrichtung sehr genau eingehalten. Die bei $22,5^{\circ}$ östlich von Süd erhöhte Zahl von Anflügen erklärt sich zwanglos : am Beobachtungstag wehte ein heftiger Ostwind, und viele Kundschafterinnen mußten durch UUberkompensation, wie sie durch v. Frisch und Lindautr $(1955,1961)$ bei Seitenwind nachgewiesen ist, in den Bereich dieses Beobachtungsplatzes gelangen. Im Anschluß an diesen Kontrolltest werden die Bienen noch 2 Tage nachdressiert und dann 3 Tage lang einem um 5 Stunden vorverschobenen Licht-Dunkel-Wechsel unterworfen. Anschließend Versetzung in ein anderes, fremdes Wiesengelände, $5 \mathrm{~km}$ vom Dressurort entfernt. Es steht der gleiche Futterplatzfächer zur Wahl. Bei kaltem, heftigem Südwestwind zeigt das gemeinsame Besuchsmaximum (Abb. 8b) aller Beobachtungsplätze eine Verstellung der inneren Uhr um 4 Std. Leider war das Wetter für die Beantwortung der zweiten, wichtigeren Frage - wird die Kompaßrichtung gemäß der verstellten Uhr gewählt, oder können die Bienen die Himmelsrichtung direkt am Sonnenstand ablesen (mit Hilfe von Azimut und Sonnenhöhe)? - nicht günstig : die Sonne war meist verdeckt, der Himmel stark bewölkt. Nach der verstellten Uhr läge die fiktive Dressurzsitmitte für die Bienen um 12.45 Uhr. Wenn die Sonnenkompaßorientierung entsprechend der inneren Uhr mit verstellt wäre, müßten die Bienen um 12.45 Uhr den gleichen Suchwinkel zwischen Sonne und Futterplatz einhalten wie vorher bei der Dressur um 16.45 Uhr; bei einem Sonnenazimut von $256,0^{\circ}$ um 16.45 Uhr wären das demnach $256,0^{\circ}-180^{\circ}=76^{\circ}$ (Abb. $9 a$ ). Um 12.45 Uhr hatte die Sonne ein Azimut von $187^{\circ}$. Ziehen wir davon die $76^{\circ} \mathrm{ab}$, dann erhalten wir die theoratische Suchrichtung von $111^{\circ}$ (Abb. 9b). Tatsächlich suchen die Bienen im Mittel bei $140^{\circ}$. D. h. die Richtungsorientierung wird nur unvollständig gemäß der verstellten inneren Uhr geändert, die starke Streuung über einen Bereich von $202,5^{\circ}$ bis $90^{\circ}$ demonstriert die Unsicherheit der Rich 


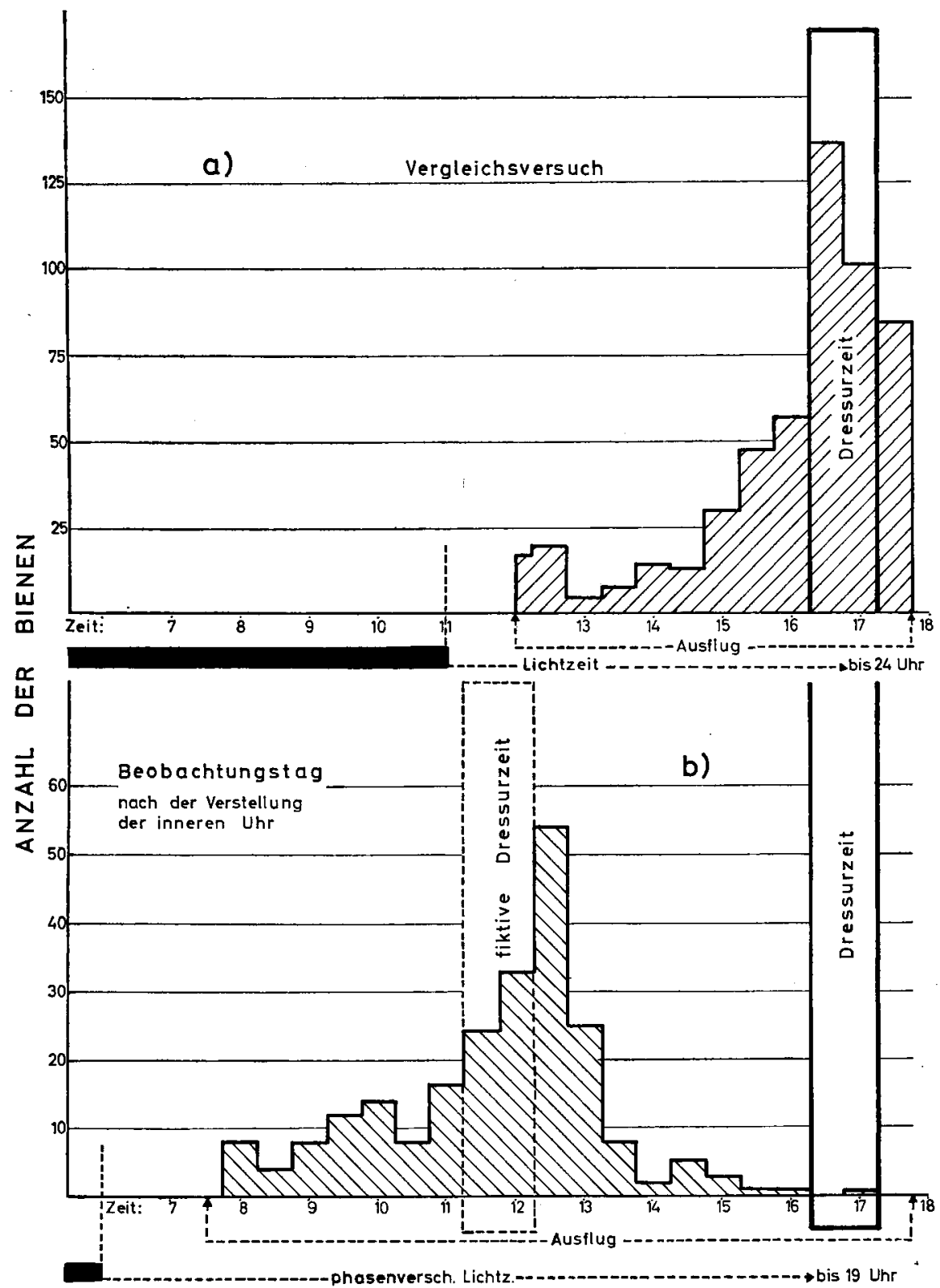

ABB. 8 (vergl. Legende zu Abb. 7)

Gleicher Versuch, bei dem jedoch die zeitliche Lage der Suchaktivität an allen Beobachtungsplätzen jeweils in einem Diagramm zusammengefaßt ist.

Fig. 8 (voir légende de la fig. 7)

Même expérience, pour laquelle cependant la position temporelle de l'activité de recherche sur l'ensemble des points d'observation a été résumée à chaque fois dans un diagramme.

bis 19 Uhr : jusqu'à 19 heures. 


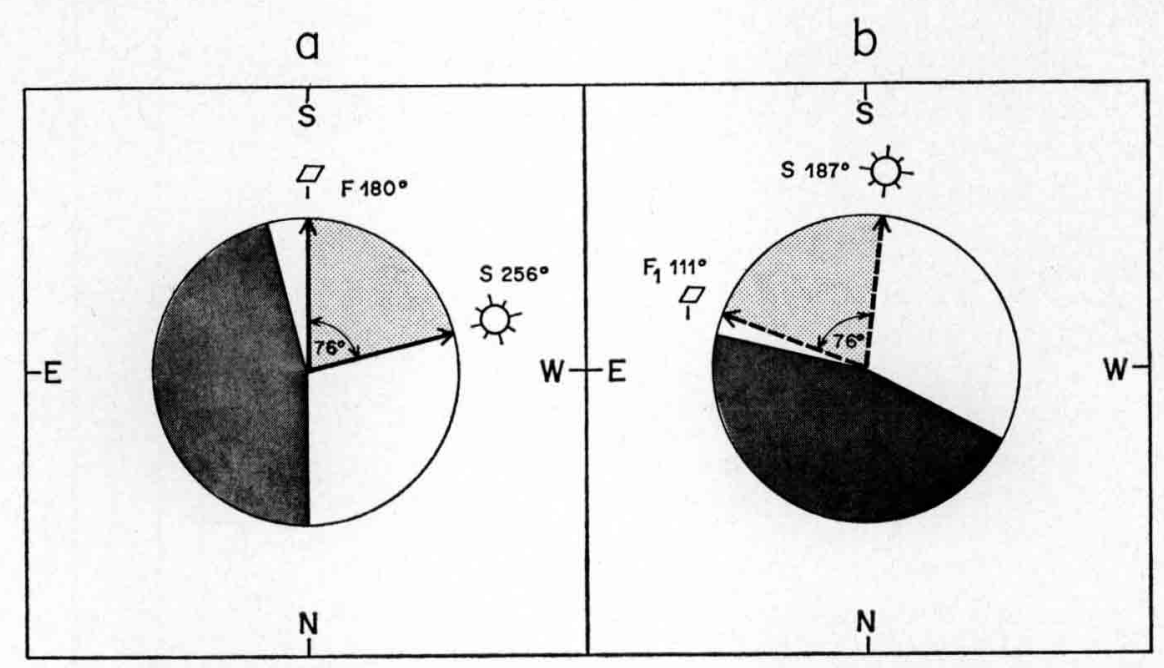

Авв. 9

Die Abb. $a$ zeigt den Winkel (grau schraffiert), den die Bienen zur Dressurzeitmitte um 16.45 Uhr zwischen Sonne und Futterplatz im Süden einhalten müssen. Dressurzeit: 16.15 - 17.15 Uhr. Die Sonne hat um 16.45 Uhr einen Azimut von $256^{\circ}$; die Differenz zum Futterplatz im Süden ergibt den Suchwinkel von $76^{\circ}$. Zur Verdeutlichung der Verstellung der inneren Uhr durch den phasenverschobenen Licht-Dunkel-Wechsel sind in $a$ und $b$ auch die jeweiligen Dunkelzeiten wiedergegeben.

Die Abb. $b$ zeigt die theoretische Suchrichtung (111\% Yu IV) der Bienen nach einer effektiven Vorverstellung der inneren Uhr um vier Stunden (Dressurzeitmitte jetzt 12.45 Uhr; Sonnenazimut 1870). $\mathrm{F}=$ Futterplatz; $\mathbf{F}_{1}=$ fiktiver Futterplatz; $\mathbf{S}=$ Sonne.

FIG. 9

a. Montre l'angle (en gris clair) que les abeilles, à l'heure moyenne de dressage (16 h 45 ), doivent maintenir entre la direction du soleil et celle du nourrisseur au sud. Heures de dressage: $16 \mathrm{~h} 15$. $17 \mathrm{~h} 15$. Le soleil a, à $16 \mathrm{~h} \mathrm{45,} \mathrm{un} \mathrm{azimut} \mathrm{de} 256^{\circ}$; la différence par rapport au nourrisseur au sud donne un angle d'exploration de $76^{\circ}$. Pour rendre plus visible le décalage de l'horloge interne par l'alternance déphasée lumière-obscurité, on a représenté en $a$ et en $b$ également à chaque fois la période d'obscurité (noir).

b. Montre la direction théorique d'exploration $\left(1^{\circ}\right)$ des abeilles après un prédécalage effectif de l'horloge interne de quatre heures (l'heure moyenne de dressage est maintenant $12 \mathrm{~h} 45$, l'azimut solaire $\left.187^{\circ}\right) . F_{1}=$ emplacement fictif du nourrisseur; $S=$ soleil.

tungswahl. Solches war bei früheren Versetzungsversuchen immer beobachtet worden, wenn der Himmel bewölkt war.

Wir führten aus diesem Grunde einen zweiten Versuch mit einem anderen Volk durch. Nach zweitägiger Umstellung auf die neue, um 5 Std. vorverstellte Licht-Dunkel-Phase ergibt sich aus den gemeinsamen Anflügen an den Beobachtungsplätzen im Mittel eine effektive Vorverstellung der inneren Uhr um 2 Std. (Abb. 10). Nach erfolgter Versetzung in fremdes Gelände fliegen die Bienen bei idealem Wetter den ganzen Tag über in so großer Zahl die Futterplätze an, daß wir kłar unsere zweite Frage beantworten können (Abb. 11). Zunächst geht eindeutig aus Abb. 11 hervor, daß die Bienen die Kompaßrichtung entsprechend der 
verstellten inneren Uhr aufsuchen. Wenn die Suchrichtung der verstellten inneren Uhr folgt, müssen wir mindestens 2 Möglichkeiten ins Auge fassen : werden die Bienen bei der Verrechnung der Sonnenwanderung eine mittlere Azimutwinkelgeschwindigkeit von $15 \%$ Std. annehmen oder werden sie eine wechselnde Azimutwinkelgeschwindigkeit berücksichtigen (vgl. v. Frisch, 1965; LindauEr, 1959)? Für den ersten Fall zeigt die Kurve $a$ in Abb. 11 die theoretische Suchrichtung am

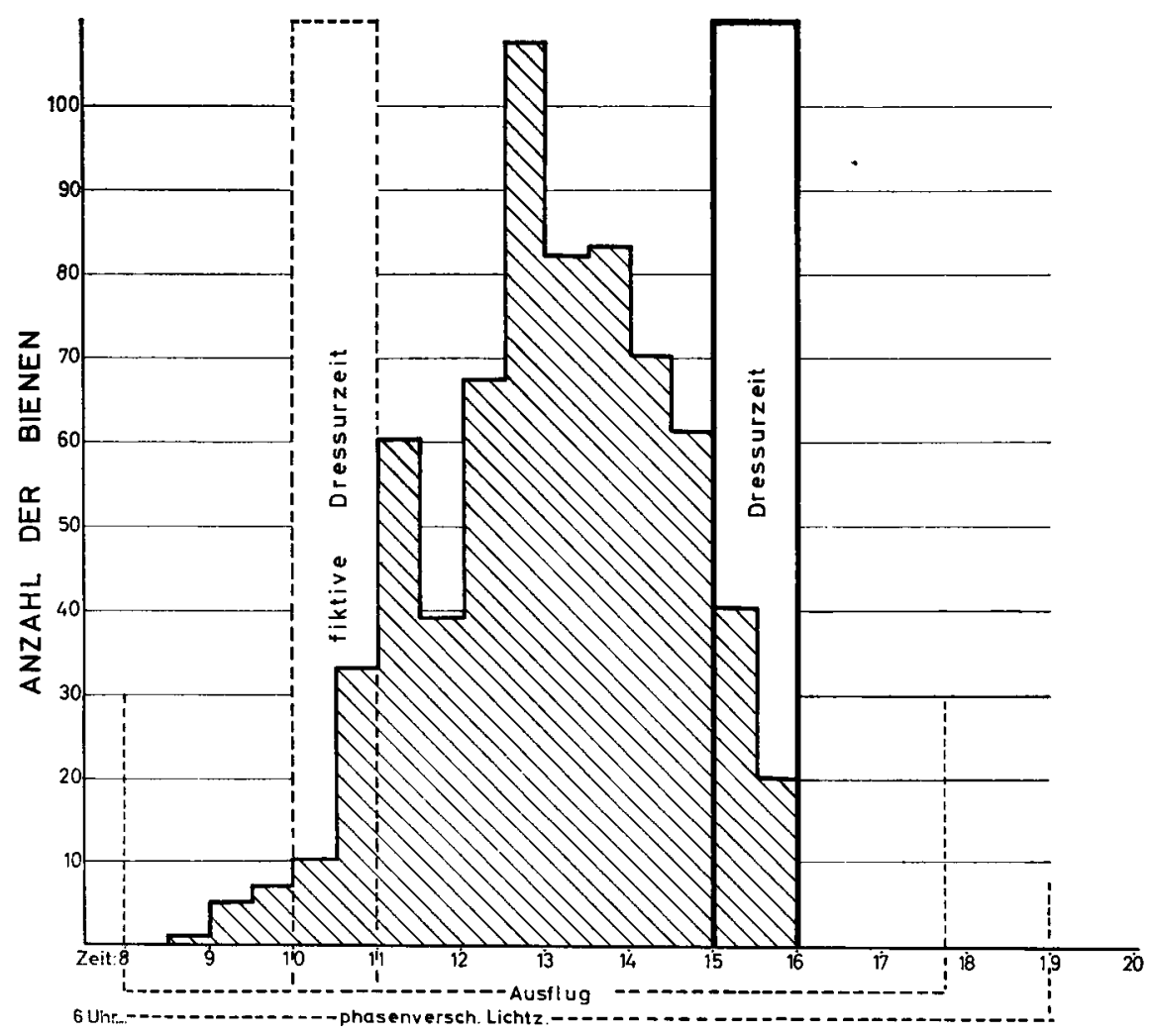

Авв. 10. - Fächer- und Versetzungsversuch bei sonnigem Wetter (vergl. Legenden zu Abb. 7 u. 8)

Das Diagramm faßt die zeitliche Lage der Suchaktivität an allen neun Beobachtungsplätzen zusammen. Vor der Beobachtung erfolgte zusammen mit der Phasenverschiebung des Licht-DunkelWechsels ( -5 Std.) eine zweitägige Arretierung der Bienen im Klimaraum.

Fig. 10. - Expérience de déplacement et expérience en êventail par temps ensoleillé (comparer avec la légende des fig. 7 et 8)

Le diagramme résume la position temporelle de l'activité exploratrice pour l'ensemble des neuf points d'observation. Avant l'observation a eu lieu en même temps que le déphasage de l'alternance lumière-obscurité ( -5 heures) une claustration de deux jours des abeilles dans la pièce climatisée. 


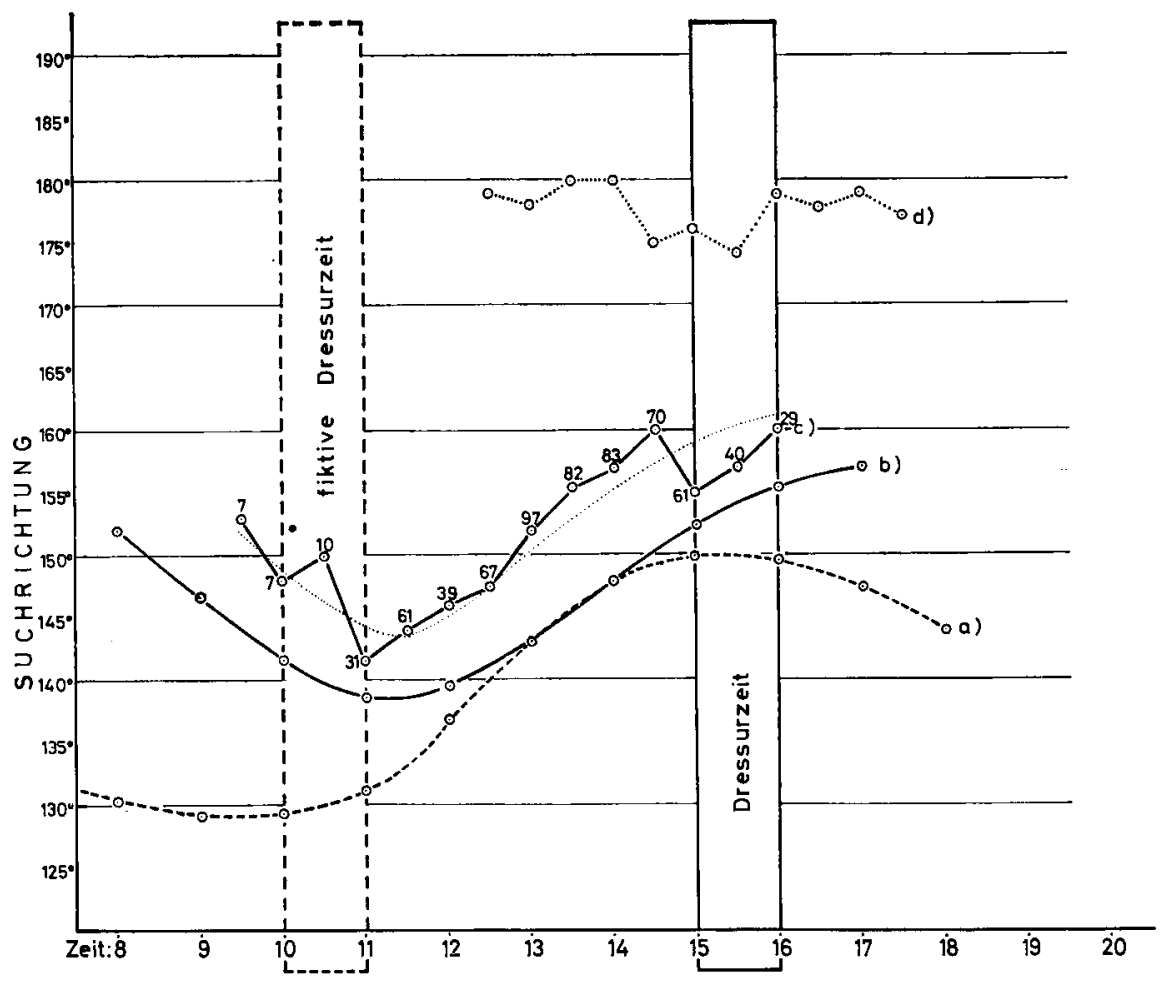

Aв8. 11. - Fächer- und Versetzungsversuch

a. Die einer tatsächlichen Vorverstellung der inneren Uhr um 2 Std. (vergl. Abb. 10) entsprechende theroretische Suchrichtung der Bienen für den Fall, dass diese den am Südplatz gelernten Winkel zur Sonne unter Berücksichtigung einer mittleren Azimutwinkelgeschwindigkeit von $15^{\circ} / \mathrm{Std}$. beibehalten.

b. Theoretische Suchrichtung der Bienen nach der Vorverstellung der inneren Uhr der Bienen um zwei Stunden unter Berücksichtigung einer variierenden Azimutwinkelgeschwindigkeit.

c. Kurve der tatsächlichen Suchrichtung der zeitdressierten Bienen. Die aus dem Diagramm ersichtliche effektive Verstellung der inneren Uhr beträgt knapp zwei Stunden. Die Zahlen bei den einzelnen Kurvenpunkten bedeuten die Suchflüge, nach denen pro halbe Stunde die Suchrichtung ermittelt wurde.

d. Tatsächliche Suchrichtung des Vergleichsversuchs vor der Phasenverschiebung.

Fıg. 11. - Expérience de déplacement et expérience en éventail

a. Direction d'exploration théorique des abeilles, correspondant à un prédécalage effectif do deux heures de l'horloge interne (voir fig. 10), dans le cas où celles-ci conservent l'angle qu'on leur a appris vers le nourrisseur sud par rapport au soleil en tenant compte d'une vitesse angulaire azimutale moyenne de $15^{\circ}$ par heure.

b. Direction d'exploitation théorique des abeilles, après le prédécalage de deux heures de l'horloge interne, en tenant compte d'une vitesse angulaire azimutale variante.

c. Courbe de la direction d'exploitation effective des abeilles dressées au temps. Le décalage effectif de l'horloge interne visible sur le diagramme est exactement de deux heures. Les nombres correspondant aux différents points de la courbe signifient les vols d'exploration d'après lesquels, par chaque demi-heure, la direction d'exploration a été calculée.

d. Direction d'exploration effective dans l'expérience témoin avant le déphasage.

Suchrichtung : direction d'exploration. 
Beobachtungstag bei zweistündiger Verschiebung, für den zweiten Fall wird die theoretische Suchrichtung durch die Kurve $b$ ausgedrückt. Die tatsächlichen mittleren Suchrichtungen aller Beobachtungsplätze ergeben die Kurve c. Sie weist bei einem geringen Phasenunterschied eine gute Übereinstimmung mit der Kurve $b$ auf. Wir müssen daraus folgern, daß die Bienen nicht die mittlere, sondern die wechselnde Azimutwinkelgeschwindigkeit der Sonne verrechnen.

Betrachten wir uns nun den Phasenunterschied zwischen Kurve $b$ und $c$. Er beträgt im Mittel $7^{\circ}$. Dies bedeutet, daß die Änderung der Richtungsorientierung etwas hinter der aus der Abb. 9 ersichtlichen Verschiebung der inneren Uhr zurückbleibt. Bei einer mittleren Azimutwinkelgeschwindigkeit von $17,4 \% / \mathrm{Std}$. zwischen 9.00 und 16.00 Uhr ist das eine zeitliche Differenz von 0,4 Std. Dieser Unterschied ist so gering, daß sich damit die größere Differenz des ersten Versuches $\left(2^{\circ}\right)$ kaum stützen läßt. Wir müssen deshalb annehmen, daß die Richtungsorientierung der Bienen beim Verstellen der inneren Uhr durch äußere Zeitgeber ebenfalls (und wahrscheinlich gleichwertig) mit verstellt wird. Zweifellos ist derselbe Zeitmeßmechanismus, der dem Zeitgedächtnis zugrunde liegt und eine Zeitdressur ermöglicht, auch für die Richtungsorientierung nach dem Sonnenkompaß verantwortlich. Das Ergebnis stimmt mit den Befunden überein, die an Staren (Hoffmann, 1954, 1960) Talitrus und Talorchestia (Pardi und Grassi, 1955) und an Tauben (Sснмidt-König, 1958, 1960, 1964) gewonnen wurden.

\section{RÉSUMÉ}

\section{INTRODUCTION}

Des travaux récents sur la mémoire du temps chez les abeilles ont montré :

$1^{0}$ Le mécanisme de mesure du temps peut être décomposé expérimentalement (par narcose ou bien par déphasage) en au moins deux composantes : l'une se manifeste comme étant le rythme circadien rigoureux, l'autre se laisse influencer par des facteurs exogènes.

$2^{\circ}$ Ainsi que de nombreux auteurs l'ont montré, le rythme circadien chez l'abeille peut être synchronisé par l'alternance journalière de la lumière et de l'obscurité. Une alternance artificielle lumière-obscurité dont la période s'éloigne de l'intervalle de 24 heures peut avoir une action synchronisatrice dans des limites étroites (de 20 à 26 heures). Dans ce cas l'avance de la période donne de meilleurs résultats que le retard.

A partir de ces données se pose la question de l'influence relative des indicateurs de temps naturels et artificiels. A ce sujet, les travaux de RENNER (1959 et 1960) ont apporté des résultats décisifs. Nous espérons, par nos expériences, répondre à la question de savoir dans quelle mesure la 
mémoire du temps des abeilles et leur faculté d'orientation par le compas solaire reposent sur le même système de mesure du temps. Cette question est importante car, en dépit des travaux de Grabensberger (1934 $a, b, c$ ) sur les Fourmis et sur les Guêpes, une mémoire du temps chez les insectes n'a pu être mise en évidence exactement que chez les abeilles. En revanche, il semble que la faculté d'orientation à partir du compas solaire soit générale chez les Arthropodes.

\section{MATÉRIEL ET MÉTHODE}

D'après les expériences antérieures (Beier, 1968, et Beier, Medugorac, Lindauer, 1968), la synchronisation de l'horloge interne des Abeilles a été obtenue par le moyen d'un indicateur de temps lumière-obscuricé, à l'intérieur d'une chambre de vol.

Dans les expériences combinées en milieu clos et à l'air libre, on a donné aux abeilles la possibilité de sortir par un sas. Ce sas permettait d'exercer un contrôle sur les heures de sortie. Il consistait en un système de sens unique commandé par des portes légères en matière plastique (voir BeIER, 1968, fig. 2).

Pour favoriser le décalage de l'horloge interne des abeilles, l'action de la phase décalée lumièreobscurité était renforcée en simulant des conditions de mauvais temps dans l'enceinte de vol (température abaissée à $+8^{\circ} \mathrm{C}$ ). Les abeilles ne volent pas à cette température. Par ailleurs, $1^{\prime}$ horloge interne se laisse diriger directement sans difficulté par le rythme de l'éclairement offert au travers d'une plaque de verre.

Le dressage sur une heure déterminée et le comptage des abeilles en expérience sont faits selon les méthodes habituelles (BeLING, 1929; W $\mathrm{W}_{\mathrm{AHL}}$, 1932; von $\mathrm{F}_{\mathrm{RISCH}}$ 1921).

\section{RÉSULTATS}

Les résultats des observations sont résumés brièvement dans les paragraphes suivants.

1. On obtient un décalage de l'horloge interne des abeilles même si, le dressage se faisant à l'extérieur, le déphasage de l'indicateur de temps exogène a été obtenu par une lumière artificielle dans une chambre climatisée. Comparativement aux expériences de déphasage avec dressage en chambre de vol, les expériences en plein air permettent nettement plus difficilement d'obtenir un décalage de l'horloge interne.

2. On explique la plus grande résistance en plein air à l'indicateur de temps artificiel lumièreobscurité déphasé, par l'influence de la position du soleil. Combinée avec des repères sur le terrain, cette position pourrait être conservée en mémoire comme schéma précis et servir ainsi d'horloge solaire.

3. Dans toutes les expériences de dressage en chambre de vol le maximum des visites a toujours lieu plus ou moins tôt avant l'heure pour laquelle a eu lieu le dressage (différence angulaire négative de phase); dans les expériences en plein air ce maximum n'apparait, pendant les jours ensoleiliés, qu'au début ou pendant l'heure pour laquelle a eu lieu le dressage alors que, lorsque le temps est pluvieux ou couvert, il a lieu cependant avant cette heure. Nous avons expliqué également la position plus précise du maximum de visites pendant les journées ensoleillées, par l'influence supplémentaire et synchronisante de l'horloge solaire.

4. Ni l'aurore ni le crépuscule n'ont d'influence sur la position du maximum de visites. 
5. Le même mécanisme de mesure du temps, qui est à l'origine de la mémoire du temps chez les abeilles et qui permet un dressage au temps, est également responsable de leur orientation directionnelle d'après le soleil.

6. Dans le calcul du déplacement apparent du soleil pour leur orientation d'après le compas solaire, les abeilles ne tiennent pas compte d'une vitesse angulaire azimutale moyenne mais de la variation de vitesse.

\section{LITERATUR}

Aschoff J. und R. Wever, 1963. Resynchronisation der Tagesperiodik von Vögeln nach Phasensprung des Zeitgebers. Z. vergl. Physiol., 46, 321-375.

BeIER W., 1968. Beeinflußung der inneren Uhr durch Phasenverschiebung des Licht-DunkelZeitgebers. Z. Bienenforsch., 9, 356-378.

Beier W., Medugorac J., und Lindauer M., 1968. Synchronisation et dissociation de "l'horloge interne" des abeilles par des facteurs externes. Ann. Epiphyties, 19, (I), 133-144.

BeLING I., 1929. Uber das Zeitgedächtnis der Bienen. Z. vergleich. Physiol., 9, 259-338.

BenNet M.-F. and Renner M., 1963. The collecting performance of Honey-bees under laboratory conditions. Biol. Bull., 125, 416-430.

Frisch K. v., 1965. Tanzsprache und Orientierung der Bienen. Springer-Verlag, Berlin-Heidelberg. New York.

Frisch K. v. und Lindauer M., 1954. Himmel und Erde in Konkurrenz bei der Orientierung der Bienen. Naturwissenschaften, 41, 245-253.

Frisch K. v. und Lindauer M., 1955. Utber die Fluggeschwindigkeit der Bienen und ihre Richtungsweisung bei Seitenwind. Naturwissenschaften, 42, 377-385.

Frisch K. v. und Lindauer M., 1961. Uber die "Mißweisung " bei den richtungsweisenden Tänzen der Bienen. Naturwissenschaften, 48, 585-594.

Hoffmann K., 1954. Versuche zu der im Richtungsfinden der Vögel enthaltenen Zeitschätzung. $Z$. Tierpsychologie 11, 453-475.

Hofrmann K., 1960. Experimental manipulations of the Orientational Clock in Birds. Cold Spring Harbor Symp. on Quant. Biol., 25.

LindaUer M., 1959. Angeborene und erlernte Komponenten in der Sonnenkompaßorientierung der Bienen. Z. vergleich. Physiol., 43, 43-62.

Lindauer M., 1963. Allgemeine Sinnesphysiologie. Orientierung im Raum. Fortschr. d. Zool. Dtsch., $16,58-140$.

PArdi L. und Grassi M., 1955. Experimental Modification of Direction-Finding in Talitrus saltator and Talorchestia deshayesii. Experimentia, Basel, 11, 202-205 (1955).

ReNner M., 1955. Ein Transozeanversuch zum Zeitsinn der Honigbienen. Naturwissenschaften, 42, 540.

Renner M., 1955. Die Haltung von Bienen in geschlossenen und künstlich beleuchteten Räumen. Naturwissenschaften 42, 539-540.

Renner M., 1957. Neue Versuche über den Zeitsinn der Honigbiene. Z. vergleich. Physiol., 40, 85-118.

Renner M., 1958. Der Zeitsinn der Arthropoden. Ergebn. Biol., 20, 127-158.

Renner M., 1959. Über ein weiteres Versetzungsexperiment zur Analyse des Zeitsinns und der Sonnenkompaßorientierung der Honigbiene. Z. vergleich. Physiol., 42, 449-483.

Renner M., 1960. The contribution of the Honeybee to the study of time-sense and astronomical orientation. in : Cold Spring Harbor Symp. on Quant. Biol. 25, 361-367.

ReNNER M., 1961. Zeitsinn und astronomische Orientierung der Honigbiene. Naturw. Rundschau, 296-305. 
SchмтDт-König K., 1958. Experimentelle Einflußnahme auf die 24-Stunden-Periodik bei Brieftauben und deren Auswirkungen unter besonderer Berücksichtigung des Heimfindevermögens. $Z$. Tierpsychol., 15, 301-331.

SchmidT-König K., 1960. Internal Clocks and Homing. Cold Spring Harbor Symp. on Quant. Biol., 25.

SchмidT-KönIG K., 1964. Über die Orientierung der Vögel. Experimente und Probleme. Naturwissenschaften, 18, 423-431.

WAHL O., 1932. Neue Untersuchungen über das Zeitgedächtnis der Bienen. Z. vergleich. Physiol., 16, 529-589.

WAHL O., 1933. Beitrag zur Frage der biologischen Bedeutung des Zeitgedächtnisses der Bienen. $Z$. vergleich. Physiol., 18, 709-717. 